\title{
Estimativa de parâmetros para um modelo de populações acopladas
}

\author{
Flávia T. Giordani ${ }^{1}$ \\ UFSC, Florianópolis, SC \\ Fermín S. V. Bazán² \\ UFSC, Florianópolis, SC \\ Luciano Bedin ${ }^{3}$ \\ UFSC, Florianópolis, SC
}

\begin{abstract}
Resumo Nesse trabalho consideramos um modelo discreto metapopulacional de $N$ sítios e de uma única espécie, tal que a dinâmica local satisfaz o modelo de Ricker e há migração de indivíduos entre os sítios. O objetivo do trabalho é a estimativa da taxa de crescimento intrínseco da população, da capacidade de suporte de cada sítio e da fração de indivíduos que migra em cada sítio, a partir de dados sintéticos que fornecem a quantidade de indivíduos em cada sítio em $\ell$ gerações. Para isso, um problema de mínimos quadrados é formulado e resolvido numericamente através de uma técnica de otimização de região de confiança com restrições de caixa.
\end{abstract}

Palavras-chave. Metapopulação, mapa de Ricker, quadrados mínimos; método da região de confiança

\section{Introdução}

Modelos metapopulacionais tem-se constituído numa importante ferramenta no estudo da degradação de ambientes naturais e da conservação das espécies [3,8,9,12]. Um dos aspectos imprescindíveis para que esses modelos forneçam previsões confiáveis a respeito do comportamento da dinâmica do sistema, é o ajuste adequado dos parâmetros biológicos envolvidos ao modelo [3,13]. A identificação de quais parâmetros devem ser incorporados ao modelo e a posterior estimativa desses parâmetros a partir de dados observacionais é uma tarefa complexa [9,12,13]. Diferentes técnicas e abordagens podem ser encontradas na literatura; por exemplo, em [10], a técnica da maximização da probabilidade é utilizada para estimar os parâmetros de sobrevivência e de migração de uma metapopulação de borboletas; em [5], a mesma técnica é utilizada, mas com a adição de efeitos de estocasticidade do meio ambiente; em [12], técnicas estatísticas baseadas em simulação Monte Carlo são utilizadas; paralelamente, em $[6,14]$, podemos encontrar técnicas de otimização aplicadas à estimativa de parâmetros em sistema de equações não-lineares.

No presente trabalho, abordamos a estimativa de parâmetros para um modelo metapopulacional discreto de uma única espécie, composto de $N$ sítios conectados por processos migratórios [7]. Baseados em [4], supomos que a dinâmica local em cada sítio é tal que a função de reprodução e sobrevivência da espécie é dada pelo mapa de Ricker [11] e que a fração $\bar{\mu}$ de indivíduos que migra em cada sítio é independente da densidade. A metapopulação é então modelada como

\footnotetext{
${ }^{1}$ flavia.giordani@ufsc.br.

${ }^{2}$ fermin.bazan@ufsc.br.

${ }^{3}$ luciano.bedin@ufsc.br.
} 
uma rede de mapas acoplados em que cada sítio pode ser interpretado como o vértice de um grafo, com a função de sobrevivência e reprodução operando em cada vértice, e as interações ocorrendo através dos efeitos dispersivos [7]. O objetivo do trabalho é apresentar a estimativa dos parâmetros $r, \kappa$ e $\bar{\mu}$, sendo $r$ a taxa de crescimento intrínseco da população e $\kappa$ a capacidade de suporte de cada sítio $[5,11,15]$, a partir de dados sintéticos que fornecem o número de indivíduos em cada sítio em $\ell$ gerações. Assim como em [4,5], os dados sintéticos são simulados a partir do sistema e subsequentemente são imbuídos de ruído, de forma a imitarem dados observacionais. O problema de estimar $r, \kappa$ e $\bar{\mu}$ é então formulado como um problema de mínimos quadrados. Estabelecendo condições necessárias para a solução do problema através da obtenção da matriz de sensitividade [9], obtemos aproximações numéricas para os parâmetros por meio de uma técnica de otimização de região de confiança com restrições de caixa [1]. Diferentemente de [4], onde os parâmetros do modelo são estimados separadamente, desconsiderando-se a conexão entre os sítios e os processos migratórios, e da abordagem SPOM (stochastic patch occupancy models [12]), que ignora a dinâmica local, nossa abordagem é mais geral, uma vez que o problema é formulado a partir da dinâmica global do sistema.

\section{Modelo}

O modelo metapopulacional no qual este trabalho está baseado consiste em uma coleção de sítios enumerados por $1,2, \ldots, N$. Os sítios correspondem a fragmentos de habitat que possuem recursos necessários à reprodução e à sobrevivência das populações que o habitam. Vamos denotar por $x_{j}^{t}$ a população total de indivíduos no sítio $j$, no tempo $t$. Cada passo de tempo corresponde a uma geração. Na ausência de migração entre os sítios, supomos que a dinâmica local é dada por $x_{j}^{t+1}=f\left(x_{j}^{t}\right), j=1,2, \ldots, N, t=0,1,2, \ldots$, sendo $f: \mathbb{R} \rightarrow \mathbb{R}$ uma função suficientemente suave que incorpora processos de reprodução e sobrevivência em cada sítio. Vamos admitir também que há conexões entre os sítios, as quais propiciam a migração de indivíduos. Uma hipótese importante no modelo é de que o processo de migração de um determinado sítio para outros sítios ocorre em cada geração após o processo de dinâmica local. Dessa forma, a cada geração, uma fração $\mu_{j i}$ de indivíduos do sítio $i$ migra para o sítio $j$, em um processo de curta duração, de forma que é razoável supormos que não há morte de indíviduos quando em migração. Por simplicidade, admitimos que os processos migratórios são independentes da densidade populacional de forma que $\mu_{j i}=c_{j i} \bar{\mu}$, sendo $0<\bar{\mu} \leq 1$ a fração de indivíduos que migra em cada sítio e $0 \leq c_{j i} \leq 1, c_{i i}=0$, a proporção dos indivíduos que migram do sítio $i$ e chegam no sítio $j$. Podemos então descrever a dinâmica metapopulacional como $[7,8]$

$$
x_{j}^{t+1}=(1-\bar{\mu}) f\left(x_{j}^{t}\right)+\sum_{i=1}^{N} c_{j i} \bar{\mu} f\left(x_{i}^{t}\right), \quad t=0,1,2, \ldots, \quad j=1,2, \ldots, N .
$$

Em (1), a expressão $c_{j i} \bar{\mu} f\left(x_{i}^{t}\right)$ representa a quantidade de indivíduos que deixam o sítio $i$ e movemse para o sítio $j$ no tempo $t$. Assim, os dois primeiros termos à direita da equação (1) representam a quantidade de indivíduos que permanece no sítio $j$ após o processo de migração, e o segundo termo representa a quantidade de indivíduos provenientes dos sítios vizinhos ao sítio $j$. Para haver consistência com a hipótese de que os processos migratórios são $100 \%$ bem sucedidos, devemos admitir que $\sum_{j=1}^{N} c_{j i}=1$, ou seja, a matriz $C=\left[c_{i j}\right]$ é do tipo coluna estocástica.

Nesse trabalho consideramos a dinâmica local determinada pelo modelo de Ricker, o qual é dado por

$$
f(u, r, \kappa)=u \exp (r(1-(u / \kappa))),
$$


sendo $r>0$ a taxa de crescimento per capita intrínseco da população, $\kappa$ a capacidade de suporte do sítio $[4,15]$ e $u$ a população local. A escolha do modelo de Ricker é de interesse puramente prático, uma vez que este tem se mostrado bastante versátil e eficaz na modelagem da dinâmica de vários tipos de organismos incluindo micróbios, peixes e insetos [4,11].

Denotando $x^{t}=\left[\begin{array}{llll}x_{1}^{t} & x_{2}^{t} & \ldots & x_{N}^{t}\end{array}\right]^{T}$, o sistema (1) pode ser reescrito como

$$
x^{t+1}=\left(I_{N}-\bar{\mu} B\right) F\left(x^{t}, r, \kappa\right), \quad t=1,2, \ldots
$$

sendo $F: \mathbb{R}^{N+2} \rightarrow \mathbb{R}^{N}$ dado por

$$
F(x, r, \kappa)=\left[\begin{array}{llll}
f\left(x_{1}, r, \kappa\right) & f\left(x_{2}, r, \kappa\right) & \ldots & f\left(x_{N}, r, \kappa\right)
\end{array}\right]^{T}, \quad x=\left(x_{1}, x_{2}, \ldots, x_{N}\right)
$$

e $B=I_{N}-C, I_{N}$ é a matriz identidade de ordem $N$.

\section{Estimativa dos parâmetros $r, \kappa$ e $\bar{\mu}$}

Vamos supor que há dados observacionais ou sintéticos disponíveis para a quantidade de indivíduos em cada sítio, para determinadas gerações $t=0,1, \ldots, \ell$. Denotemos por $\widetilde{x}_{j}^{t}$ essa quantidade de indivíduos referente ao sítio $j$ e geração $t$ e definimos $\widetilde{x}^{t}=\left[\begin{array}{llll}\widetilde{x}_{1}^{t} & \widetilde{x}_{2}^{t} & \ldots & \widetilde{x}_{N}^{t}\end{array}\right]^{T}$. Do ponto de vista prático, admitindo que conhecemos a matriz $C$, devemos encontrar os parâmetros $r$, $\kappa$ e $\bar{\mu}$ adequados de forma que o sistema (3) descreva da forma mais apurada possível a dinâmica da metapopulação. Para isso, consideramos como condição inicial para o sistema (3) o vetor $\widetilde{x}^{0}$, sendo que os demais $x^{t}$ são obtidos iterando-se esse sistema. Notamos que nesse processo, $x^{t}$ corresponde a uma expressão dada em termos de $r, \kappa$ e $\bar{\mu}$, uma vez que, nesse estágio, esses parâmetros são desconhecidos. Queremos encontrar $(\bar{\mu}, \kappa, r)$ que melhor se ajusta ao sistema, em outras palavras temos de resolver o seguinte problema de minimização com restrições [14]:

$$
\min _{(\bar{\mu}, \kappa, r) \in K} H(\bar{\mu}, r, \kappa),
$$

sendo

$$
H(\bar{\mu}, r, \kappa)=\frac{1}{2} \sum_{t=1}^{\ell}\left|x^{t}(\bar{\mu}, r, \kappa)-\widetilde{x}^{t}\right|^{2} ;
$$

e $K=\left[\mu_{1}, \mu_{2}\right] \times\left[\kappa_{1}, \kappa_{2}\right] \times\left[r_{1}, r_{2}\right]$; os limitantes $0 \leq \mu_{1}<\mu_{2} \leq 1,0<\kappa_{1}<\kappa_{2}, 0<r_{1}<r_{2}$ são dados previamente. A existência de soluções para o problema (5) é uma consequência direta do Teorema de Weierstrass, uma vez que $x^{t}, t=0,1, \ldots, \ell$, é uma composição de um número finito de funções suaves. Por outro lado, a solução pode não ser única e temos de lidar com a possível existência de múltiplos mínimos locais.

Uma condição necessária para a existência de um mínimo local $(\bar{\mu}, r, \kappa)$ de $H$ é que $\nabla H(\bar{\mu}, r, \kappa)=$ $(0,0,0)$. Sendo assim, derivando o funcional com relação a cada um dos parâmetros e igualando a zero obtemos

$$
\sum_{t=1}^{\ell} \sum_{i=1}^{N}\left(x_{i}^{t}-\widetilde{x}_{i}^{t}\right) \frac{\partial x_{i}^{t}}{\partial \bar{\mu}}=0, \quad \sum_{t=1}^{\ell} \sum_{i=1}^{N}\left(x_{i}^{t}-\widetilde{x}_{i}^{t}\right) \frac{\partial x_{i}^{t}}{\partial r}=0, \quad \sum_{t=1}^{\ell} \sum_{i=1}^{N}\left(x_{i}^{t}-\widetilde{x}_{i}^{t}\right) \frac{\partial x_{i}^{t}}{\partial \kappa}=0 .
$$

Agora, derivando (3) com respeito a $\bar{\mu}, r$ e $\kappa$, obtemos

$$
\begin{aligned}
\frac{\partial x^{t+1}}{\partial \bar{\mu}} & =-B F\left(x^{t}, r, \kappa\right)+\left(I_{N}-\bar{\mu} B\right) \frac{\partial F}{\partial u}\left(x^{t}, r, \kappa\right) \frac{\partial x^{t}}{\partial \bar{\mu}}, \quad t=1,2, \ldots \\
\frac{\partial x^{t+1}}{\partial r} & =\left(I_{N}-\bar{\mu} B\right)\left[\frac{\partial F}{\partial r}\left(x^{t}, r, \kappa\right) \cdot \mathbf{1}_{N}+\frac{\partial F}{\partial u}\left(x^{t}, r, \kappa\right) \frac{\partial x^{t}}{\partial r}\right], \quad t=1,2, \ldots \\
\frac{\partial x^{t+1}}{\partial \kappa} & =\left(I_{N}-\bar{\mu} B\right)\left[\frac{\partial F}{\partial \kappa}\left(x^{t}, r, \kappa\right) \cdot \mathbf{1}_{N}+\frac{\partial F}{\partial u}\left(x^{t}, r, \kappa\right) \frac{\partial x^{t}}{\partial \kappa}\right], \quad t=1,2, \ldots
\end{aligned}
$$


em que

$$
\begin{aligned}
& \frac{\partial F}{\partial u}\left(x^{t}, r, \kappa\right)=\operatorname{diag}\left(\frac{\partial f}{\partial u}\left(x_{1}^{t}, r, \kappa\right), \frac{\partial f}{\partial u}\left(x_{2}^{t}, r, \kappa\right), \ldots, \frac{\partial f}{\partial u}\left(x_{N}^{t}, r, \kappa\right)\right) \\
& \frac{\partial F}{\partial r}\left(x^{t}, r, \kappa\right)=\operatorname{diag}\left(\frac{\partial f}{\partial r}\left(x_{1}^{t}, r, \kappa\right), \frac{\partial f}{\partial r}\left(x_{2}^{t}, r, \kappa\right), \ldots, \frac{\partial f}{\partial r}\left(x_{N}^{t}, r, \kappa\right)\right) \\
& \frac{\partial F}{\partial \kappa}\left(x^{t}, r, \kappa\right)=\operatorname{diag}\left(\frac{\partial f}{\partial \kappa}\left(x_{1}^{t}, r, \kappa\right), \frac{\partial f}{\partial \kappa}\left(x_{2}^{t}, r, \kappa\right), \ldots, \frac{\partial f}{\partial \kappa}\left(x_{N}^{t}, r, \kappa\right)\right)
\end{aligned}
$$

e $\mathbf{1}_{N}=\left[\begin{array}{lllll}1 & 1 & 1 & \ldots & 1\end{array}\right]^{T}$. O sistema (8)-(10) é complementado com as condições iniciais $\frac{\partial x^{0}}{\partial \bar{\mu}}=0$, $\frac{\partial x^{0}}{\partial r}=0$ e $\frac{\partial x^{0}}{\partial \kappa}=0$. Definindo

$$
\mathbf{J}(\bar{\mu}, r, \kappa)=\left[\begin{array}{lll}
\frac{\partial x^{1}}{\partial \bar{\mu}} & \frac{\partial x^{1}}{\partial r} & \frac{\partial x^{1}}{\partial \kappa} \\
\frac{\partial x^{2}}{\partial \bar{\mu}} & \frac{\partial x^{2}}{\partial r} & \frac{\partial x^{2}}{\partial \kappa} \\
\vdots & \vdots & \vdots \\
\frac{\partial x^{\ell}}{\partial \bar{\mu}} & \frac{\partial x^{\ell}}{\partial r} & \frac{\partial x^{\ell}}{\partial \kappa}
\end{array}\right], \quad \mathbf{X}(\bar{\mu}, r, \kappa)=\left[\begin{array}{l}
x^{1}(\bar{\mu}, r, \kappa) \\
x^{2}(\bar{\mu}, r, \kappa) \\
\vdots \\
x^{\ell}(\bar{\mu}, r, \kappa)
\end{array}\right], \quad \widetilde{\mathbf{X}}=\left[\begin{array}{l}
\tilde{x}^{1} \\
\widetilde{x}^{2} \\
\vdots \\
\tilde{x}^{\ell}
\end{array}\right],
$$

as equações (7) podem ser reescritas como

$$
\mathbf{J}^{T}(\bar{\mu}, r, \kappa)(\mathbf{X}(\bar{\mu}, r, \kappa)-\widetilde{\mathbf{X}})=0 .
$$

Em outras palavras, (11) é uma condição necessária para que $H$ tenha um mínimo local em $(\bar{\mu}, r, \kappa)$. $\mathrm{Na}$ literatura, $\mathbf{J}$ é referida como a matriz de sensitividade $[3,9]$.

\section{Resultados Numéricos}

Para ilustrar o método, geramos dados sintéticos para o problema baseando-nos em procedimento adotado em [4]: dados são obtidos de forma a simular, tão realisticamente quanto possível, resultados experimentais obtidos a partir de uma rede de $N=9$ subpopulações do inseto Drosophila, dispostas ao longo da borda de um círculo. Os insetos em cada sítio podem migrar para os dois sítios mais próximos, de forma que a matriz $C$ é dada por

$$
C=\left[\begin{array}{llllllll}
0 & 0,5 & 0 & 0 & 0 & \ldots & 0 & 0,5 \\
0,5 & 0 & 0,5 & 0 & 0 & \ldots & 0 & 0 \\
0 & 0,5 & 0 & 0,5 & 0 & \ldots & 0 & 0 \\
\vdots & \vdots & \vdots & \vdots & \vdots & \vdots & \vdots & \vdots \\
0 & 0 & 0 & \ldots & 0 & 0.5 & 0 & 0.5 \\
0,5 & 0 & 0 & \ldots & 0 & 0 & 0,5 & 0
\end{array}\right]
$$

A condição inicial em cada sítio é $\widetilde{x}_{i}^{0}=20$ para $1 \leq i \leq 5, \widetilde{x}_{i}^{0}=21$ para $6 \leq i \leq 9$.

O método usado na estimativa dos parâmetros de interesse é baseado numa técnica de otimização de região de confiança com restrições de caixa, conforme descrito no trabalho clássico de Coleman e Li [1], e disponível no MATLAB (m-file lsqnonlin). Para completude, notamos que a minimização de $H(p), p=(\bar{\mu}, r, \kappa)$, por meio de um método de região de confiança consiste em construir uma sequência de aproximações $p^{(j)}$ onde o incremento $s^{(j)}=p^{(j+1)}-p^{(j)}$ é solução do subproblema quadrático com limitante sobre o incremento,

$$
\min _{s \in \mathbb{R}^{3}}\left\{q_{j}(s)=g_{j}^{T} s-\frac{1}{2} s^{T} B_{j} s,\|s\| \leq \Delta_{k}\right\},
$$


em que $g_{j}$ denota o gradiente de $H$ descrito em (11) no ponto $p^{(j)}$ e $B_{j}$ é uma matriz simétrica que aproxima a Hessiana de $H$, por exemplo $\mathbf{J}^{T}\left(p^{(j)}\right) \mathbf{J}\left(p^{(j)}\right)$. Uma abordagem compreensiva sobre métodos de região de confiança pode ser encontrada em [2].

Apresentamos resultados numéricos da estimativa dos parâmetros usando dados inexatos construídos a partir dos estados $x^{t}$, descritos na forma $\widetilde{x}^{t}=x^{t}+\varepsilon^{t}, t=0, \ldots, \ell$, em que $\varepsilon^{t}$ um vetor randômico de perturbações com distribuição uniforme tal que,

$$
\left\|\widetilde{x}^{t}-x^{t}\right\|=\mathrm{NL}\left\|x^{t}\right\|,
$$

e NL denota o nível de ruído nos dados. Em todos os casos, na estimativa dos parâmetros $\bar{\mu}, r$ e $\kappa$, o método é inicializado com valores randômicos. Uma vez que trabalhamos com dados sintéticos, podemos escolher previamente os valores de $\bar{\mu}, r$ e $\kappa$ a serem estimados. Com isso, o conjunto $K$ pode ser adequadamente escolhido de forma a conter todos os $(\bar{\mu}, \kappa, r)$ utilizados na simulação numérica. Utilizando essa metodologia e tendo em vista os valores dos parâmetros utilizados nas simulações apresentadas a seguir, consideramos $0,05 \leq \bar{\mu} \leq 0,9,1 \leq r \leq 5$ e $10 \leq \kappa \leq 35$.

Nas Tabelas 1-4 estimativas relativas a quatro situações típicas de interesse biológico são apresentadas. Nas colunas 2,3 e 4 de cada tabela os valores estimados de $r, \bar{\mu}$ e $\kappa$ são apresentados, enquanto que nas colunas 5, 6 e 7 constam os respectivos erros relativos, os quais denotamos por $E(r), E(\bar{\mu})$ e $E(\kappa)$. Os valores de $r$ e $\kappa$ escolhidos para ilustrar os resultados são típicos de experimentos em laboratório [4].

Tabela 1: Estimativas dos parâmetros $(\bar{\mu}, \kappa, r)$ a partir de dados com ruído para vários valores de NL. Neste caso, os dados simulados usam $r=3,8, \bar{\mu}=1 / 3$ e $\kappa=25$.

\begin{tabular}{|c|ccc||ccc|}
\hline$N L$ & $r$ & $\bar{\mu}$ & $\kappa$ & $\mathrm{E}(r)$ & $\mathrm{E}(\bar{\mu})$ & $\mathrm{E}(\kappa)$ \\
\hline 0,001 & 3,7989 & 0,3388 & 25,0024 & 0,0003 & 0,0163 & 0,0001 \\
0,0050 & 3,8035 & 0,3692 & 25,0142 & 0,0009 & 0,1075 & 0,0006 \\
0,0100 & 3,8097 & 0,3342 & 25,0235 & 0,0026 & 0,0026 & 0,0009 \\
0,1000 & 3,8126 & 0,3283 & 24,9331 & 0,0033 & 0,0151 & 0,0027 \\
0,5000 & 5,0000 & 0,9000 & 23,9443 & 0,3158 & 1,7000 & 0,0422 \\
\hline
\end{tabular}

Tabela 2: Estimativas dos parâmetros $(r, \bar{\mu}, \kappa)$ a partir de dados com ruído para vários valores de NL. Neste caso, os dados simulados usam $r=3,8, \bar{\mu}=0,7$ e $\kappa=25$.

\begin{tabular}{|c|ccc||ccc|}
\hline$N L$ & $r$ & $\bar{\mu}$ & $\kappa$ & $\mathrm{E}(r)$ & $\mathrm{E}(\bar{\mu})$ & $\mathrm{E}(\kappa)$ \\
\hline 0,0010 & 3,7990 & 0,7121 & 24,9987 & 0,0003 & 0,0173 & 0,0001 \\
0,0050 & 3,7956 & 0,7079 & 25,0083 & 0,0011 & 0,0113 & 0,0003 \\
0,0100 & 3,8222 & 0,6373 & 25,0064 & 0,0059 & 0,0896 & 0,0003 \\
0,1000 & 3,9344 & 0,9000 & 25,2355 & 0,0354 & 0,2857 & 0,0094 \\
0,5000 & 4,9753 & 0,0500 & 24,4248 & 0,3093 & 0,9286 & 0,0230 \\
\hline
\end{tabular}

Na Tabela 1 consideramos um valor relativamente baixo da taxa de migração $\bar{\mu}$, como reportado em [4]; já na Tabela 2, exploramos a estimativa dos parâmetros no caso de uma taxa de migração de maior magnitude. Na Tabela 3, consideramos o caso em que a taxa de crescimento intrínseco da população é relativamente baixa, enquanto que na Tabela 4 aumentamos a capacidade de suporte de cada sítio. Os resultados foram obtidos utilizando-se $\ell=5$. Resultados obtidos com outros valores de $\ell$, não apresentados no manuscrito devido à limitação de espaço, mostraram estimativas relativamente estáveis, dependendo do nível de ruído nos dados. Mais precisamente, para os casos $\ell=3$ ou $\ell=4$, os resultados são comparáveis aos obtidos com $\ell=5$, para pequenos valores de NL; 
no entanto, à medida que NL aumenta, os respectivos erros relativos são maiores comparativamente aos resultados do caso $\ell=5$. No outro extremo, para os valores $\ell=9$ ou $\ell=12$, o método produz resultados acurados apenas para o caso de dados com níveis de ruído superiores a 0,05\%. De modo geral, observamos que o método fornece resultados com erros relativos compatíveis com o nível de ruído respectivo, independentemente do fato de considerarmos taxas de migração baixas ou altas, ou das variações de $r$ e $\kappa$, embora isso tenha de ser investigado mais a fundo. É importante observar que quando alguma das variáveis estimadas atinge uma das cotas, superior ou inferior, o erro relativo da solução tende a aumentar. Esse é o caso, por exemplo, da Tabela 1 em que, no caso de $50 \%$ de ruído, $\bar{\mu}$ atinge a cota superior 0,9 ; o mesmo ocorre na Tabela 2 para o caso de $50 \%$ de ruído, em que o parâmetro $\bar{\mu}$ atinge a cota inferior 0,05 .

Tabela 3: Estimativas dos parâmetros $(r, \bar{\mu}, \kappa)$ a partir de dados com ruído para vários valores de NL. Neste caso, os dados simulados usam $r=2,5, \bar{\mu}=1 / 3$ e $\kappa=25$.

\begin{tabular}{|c|ccc||ccc|}
\hline$N L$ & $r$ & $\bar{\mu}$ & $\kappa$ & $\mathrm{E}(r)$ & $\mathrm{E}(\bar{\mu})$ & $\mathrm{E}(\kappa)$ \\
\hline 0,0010 & 2,5000 & 0,3473 & 24,9938 & 0,0000 & 0,0418 & 0,0002 \\
0,0050 & 2,5003 & 0,2808 & 24,9931 & 0,0001 & 0,1576 & 0,0003 \\
0,0100 & 2,5027 & 0,2623 & 24,9814 & 0,0011 & 0,2130 & 0,0007 \\
0,1000 & 2,5135 & 0,2166 & 24,6571 & 0,0054 & 0,3501 & 0,0137 \\
0,5000 & 2,9687 & 0,0500 & 23,4036 & 0,1875 & 0,8500 & 0,0639 \\
\hline
\end{tabular}

Tabela 4: Estimativas dos parâmetros $(r, \bar{\mu}, \kappa)$ a partir de dados com ruído para vários valores de NL. Neste caso, os dados simulados usam $r=3,8, \mu=1 / 3$ e $\kappa=27$.

\begin{tabular}{|c|ccc||ccc|}
\hline$N L$ & $r$ & $\bar{\mu}$ & $\kappa$ & $\mathrm{E}(r)$ & $\mathrm{E}(\bar{\mu})$ & $\mathrm{E}(\kappa)$ \\
\hline 0,0010 & 3,8010 & 0,3337 & 26,9974 & 0,0003 & 0,0011 & 0,0001 \\
0,0050 & 3,8039 & 0,3345 & 26,9897 & 0,0010 & 0,0036 & 0,0004 \\
0,0100 & 3,7970 & 0,3317 & 27,0081 & 0,0008 & 0,0048 & 0,0003 \\
0,1000 & 3,5650 & 0,1685 & 27,6562 & 0,0618 & 0,4945 & 0,0243 \\
0,5000 & 3,5124 & 0,3713 & 27,8490 & 0,0757 & 0,1140 & 0,0314 \\
\hline
\end{tabular}

\section{Conclusões}

Neste trabalho utilizamos um método de otimização de região de confiança com restrições de caixa para a estimativa de parâmetros para um modelo de populações acopladas. A estimativa é feita a partir de dados sintéticos gerados através de iterações do sistema. No caso de dados sem ruído, as soluções tem erros relativos pequenos se consideramos poucos estágios de tempo, por exemplo, $3 \leq \ell \leq 5$; utilizando dados com mais estágios de tempo, o erro relativo tende a aumentar. Uma possível justificativa para isso pode ser que o aumento do grau de sobredeterminação do sistema acarreta na existência de multiplos mínimos locais para $H$, embora essa afirmação tenha de ser investigada mais a fundo. No caso de dados com ruído, o método apresenta melhores resultados quando consideramos um número maior de estágios de tempo. A escolha de quantos e quais estágios de tempo devem ser utilizados na estimativa de parâmetros em modelos metapopulacionais é um tema controverso $[12,13]$ o qual está fora do escopo do presente trabalho. Entretanto, destacamos que, para a faixa de valores de $r$ e $\kappa$ aqui considerados, a dinâmica local obtida a partir do mapa de Ricker apresenta um comportamento complexo com a presença de órbitas caóticas, isto é, órbitas sensíveis a dados iniciais [11]. Isso pode levar o sistema (3) a ter longos transientes [4], portanto considerar dados com poucos níveis de tempo pode afetar a qualidade dos parâmetros 
estimados. De qualquer forma, a abordagem e o método se mostraram promissores, em parte devido à generalidade considerada, uma vez que a dinâmica local bem como a migração entre os sítios são incorporadas à formulação do problema. Pretendemos futuramente investigar a incorporação de efeitos de estocasticidade demográfica à abordagem [5], com o intuito de estender o método à estimação de parâmetros a partir de dados observacionais.

\section{Referências}

[1] Coleman, T. F. and Li, Y. An Interior, Trust Region Approach for Nonlinear Minimization Subject to Bounds, SIAM Journal on Optimization, 6:418-445, 1996. DOI:10.1137/0806023.

[2] Conn, A. R., Gould, N. I. M. and Toint, P. L. Trust region methods. SIAM, Philadelphia, 2000.

[3] Conroy, M. J., Cohen, Y., James, F. C., Matsinos, Y. C. and Maurer, B. A. Parameter for spatially estimation, reliability and model improvement explicit models of animal populations, Ecological Applications, 5:17-19, 1994. DOI:10.2307/1942047.

[4] Dey, S. and Joshi, A. Local Perturbations Do Not Affect Stability of Laboratory Fruitfly Metapopulations, PLoS ONE, 2:1-9, 2007. DOI:10.1371/journal.pone.0000233.

[5] Drake, J. M. Density-Dependent Demographic Variation Determines Extinction Rate of Experimental Populations, PLoS Biology, 3:1300-1304, 2005. DOI:10.1371/journal.pbio.0030222.

[6] Gábor, A. and Banga, J. R., Robust and efficient parameter estimation in dynamic models of biological systems, BMC Systems Biology, 9:1-25, 2015. DOI:10.1186/s12918-015-0219-2.

[7] Giordani, F. T. Migração dependente da densidade em modelos metapopulacionais, Tese de Doutorado, UFRGS, 2008.

[8] Hanski, I. Metapopulation Models, Encyclopedia of Ecology, Elsevier, volume 3, pages 23182325, 2008.

[9] Hanski, I., Kuussaari, M. and Nieminen, M. Metapopulation Structure and Migration in the Butterfly Melitaea Cinxia, Ecology, 75:747-762, 1994. DOI:10.2307/1941732.

[10] Hanski, I., Alho, J. and Moilanen, A. Estimating the Parameters of Survival and Migration of Individuals in Metapopulations, Ecology, 81(2000) 239-251. DOI:10.2307/177147.

[11] Ives, A. R., Woody, S. T., Nordheim, E. V., Nelson, C. and Andrews, J. H., The synergistic effects of stochasicity and dispersal on population dynamics, American Naturalist, 163:375387, 2004. DOI: 10.1086/381942.

[12] Moilanen, A. The equilibrium assumption in estimating the parameters of metapopulation models, Journal of Animal Ecology, 69:143-153, 2000. DOI:10.1046/j.1365-2656.2000.00381.x.

[13] Moilanen, A. Implications of empirical data quality to metapopulation model parameter estimation and application, Oikos, 96:516-530, 2002. DOI:10.1034/j.1600-0706.2002.960313.x.

[14] Schittkowski, K. Parameter estimation in systems of nonlinear equations, Numerische Mathematik, 68:129-142, 1994. DOI:10.1007/s002110050052.

[15] Zhang, B., DeAngelis, D. L. and Ni, W. M. Carrying Capacity of Spatially Distributed Metapopulations, Trends in Ecology \& Evolution, 36:164-173, 2020. DOI:10.1016/j.tree.2020.10.007. 